\title{
Profile of adult patients admitted with drug-induced liver injury at a district hospital in Pietermaritzburg, KwaZulu-Natal
}

\author{
K Naicker* and S Rangiah \\ Department of Family Medicine, University of KwaZulu Natal, Pietermaritzburg, Durban \\ ${ }^{*}$ Corresponding author, email: kumeshneenaicker@live.co.za
}

Background: Drug-induced liver injury (DILI) can be prevented if diagnosed and treated timeously. The identification and primary prevention of DILI risk factors presents the rational means of reducing hospital costs and mortality.

Methods: A retrospective chart review was conducted of clinical in-patient records of all adult patients admitted to Northdale Hospital (NDH) with a diagnosis of DILI. Patients with pre-existing liver disease were excluded.

Results: A total of 95 patient files with DILI were reviewed. The burden of DILI at NDH over the two-year period was $0.19 \%$. The average age was 38 years, with a slightly higher female preponderance (62.1\%). A lower serum albumin level (mean $21.35 \mathrm{~g} / \mathrm{dl}$ ) was significantly associated with DILI $(p<0.001)$. Forty-six patients had a history of alcohol consumption, which increased the risk of DILI (OR 2.1).

Of the patients reviewed, 62 (65\%) were on antiretroviral therapy (ART) whereas 41 (43\%) were on tuberculosis treatment (TBT) at presentation. The most common co-morbidities associated with DILI were HIV (75.7\%), TB (43.2\%), renal disease (34.7\%) and malnutrition (31.6\%). The most common hepatotoxins, apart from ART and TBT, were paracetamol (46.3\%), cotrimoxazole (32.6\%), alcohol (48.4\%) and traditional medication (27.4\%).

Conclusion: This study demonstrated associations with the development of DILI and being female gender, younger age group, hypoalbuminaemia and renal failure. The use of alcohol, traditional medication and the overzealous use and prescription of paracetamol to patients who present with DILI is concerning. The case fatality rate of $14.7 \%$ demonstrates the importance of identifying these potential risk factors timeously.

Keywords: drug-induced liver injury, hepatitis, HIV, jaundice

\section{Background}

In the last six years the National Department of Health has made some positive changes in improving the well-being of South Africans infected with human immunodeficiency virus (HIV). ${ }^{1,2}$ These changes included the routine use of isoniazid preventive therapy (IPT) for all people living with HIV and AIDS (PLWHA), ${ }^{1-3}$ the introduction of fixed dose combination (FDC) antiretrovirals (ARV) in 2013, and the new amended ARV 'Test and Treat' Guidelines of 2016 making access to antiretroviral therapy (ART) simpler, cheaper and more convenient. ${ }^{4}$

An estimated $65 \%$ of new tuberculosis (TB) cases in South Africa (SA) are co-infected with HIV. ${ }^{5,6}$ Upscaling ARV and tuberculosis treatment (TBT) access is largely beneficial to the greater community but still produces potential risk and complications of shared drug toxicities. ${ }^{1,7}$ A study conducted at a secondary hospital in Cape Town, South Africa, concluded that the threemonth mortality associated with ARV and TBT-related drug induced liver injury (DILI) was as high as $30 \% .{ }^{8}$ To our knowledge a risk factor study on DILI has yet not been undertaken at a district-level hospital in South Africa.

Northdale Hospital (NDH) is the only district hospital in the Msunduzi subdistrict of KwaZulu-Natal. It has a total of 385 beds in use. This hospital serves a largely disadvantaged population of 980000 and this number accounts for $60 \%$ of the total population in the uMgungundlovu district. ${ }^{9}$ The district had a high HIV prevalence of $40.7 \%$ in the $2013 / 14$ financial year with HIV contributing to $8.6 \%$ of deaths. ${ }^{9}$ The TB incidence was 776/100 000 in year 2013/14 with a TB death rate of $6.7 \%{ }^{9}$
Given the burden of HIV/TB amongst the patients in our study setting, we were interested in exploring other risk factors in patients who present with DILI to NDH. Therefore, the aim of the study was to describe the profile and frequency of risk factors of patients admitted with both clinical (symptoms of hepatitis) and laboratory diagnosed DILI (Alanine aminotransferase $[\mathrm{ALT}]>120 \mathrm{IU} / \mathrm{l}$ in a symptomatic patient [nausea, vomiting, anorexia, jaundice and/ or abdominal pain], OR ALT > $200 \mathrm{IU} / \mathrm{I}$ in a well patient [asymptomatic] OR total serum bilirubin concentration [TBR] > $40 \mathrm{UM} / \mathrm{l})$. $^{1,2}$ The upper limit of normal (ULN) for ALT and TBR as per the SA National Health Laboratory Service (NHLS) reference range was $40 \mathrm{lU} / \mathrm{l}$ and $20 \mathrm{UM} / \mathrm{l}$, respectively.

\section{Methods}

This was a retrospective descriptive study performed at NDH. All records of suitable medical patients above 18 years who were admitted to the medical wards with a clinical and laboratory diagnosis of DILI during the two-year study period were reviewed (Figure 1). In total, 95 cases were included. Thirtyseven cases that were excluded did not meet the criteria for DILI (e.g. pre-existing medical and surgical hepatic disease, e.g. liver cirrhosis, cancer and pregnancy).

Relevant data were extracted using a pre-defined data collection tool. Patient records were identified using the ICD10 code K72.6 (hepatitis) in the hospital inpatient register. Further review of clinical records and laboratory data (accessed from the NHLS database) was done in order to extract the relevant parameters for this study (demographic data, clinical symptoms and signs, laboratory results, drug and co-morbidity history, and deaths). 
- Total number of patient admissions to hospital over two-year period

50139

- Total number of patient admissions to hospital for drug-induced liver injury

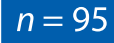

- Burden of drug-induced liver injury at $0.19 \%$ $\mathrm{NDH}$

Figure 1: Burden of DILI at NDH according to the chart review of inpatient records over the two-year period.

Statistical analysis was done using computer software (SPSS version 25, IBM Corp, Armonk, NY, USA). Data were analysed by chi-square test and logistic regression analysis. Data were expressed as 'mean (standard deviation; SD)', minimummaximum $95 \% \mathrm{Cl}$ and percentage. Where appropriate a $p$ value $<0.05$ was considered statistically significant. Binary logistic regression was used to calculate the adjusted odds ratio for the significant risk factors of drug-induced liver injury. Multivariate logistic regression analysis was done to remove confounding variables to assess the role of independent risk factors for development of drug-induced liver injury.

\section{Aims}

The aim of this study was to describe the clinical and demographic characteristics of patients with DILI and to identify the risk factors and in-hospital mortality associated with this disease, at Northdale Hospital (NDH), a District Hospital in KwaZulu-Natal.

\section{Ethical consideration}

Ethical approval was obtained from the University of KwaZuluNatal Biomedical Research Ethics Committee (Ref [BE008/18]) as well as permission from the KZN Department of Health and hospital management to conduct this study.

\section{Results}

A total of 95 suitable in-patient files with DILI were reviewed (Figure 1, Table 1).

The average length of hospital stay was $8.4 \pm 4.4$ days, and this stay increased to 9.9 days in DILI complicated by HIV/TB. The mean age was 38 years $(95 \% \mathrm{Cl} 35-41$ years) with a predominant female preponderance of $62 \%$. The odds of females being diagnosed with DILI was OR 1.8 and $p<0.02$. Of all the females with DILI, $90 \%$ had either HIV/TB or both, compared with $66.6 \%$ of males.

Clinically all patients had jaundice with other concomitant symptoms including nausea, vomiting, abdominal pain and diarrhoea. Of the 95 patients, 11 had encephalopathy.

A lower serum albumin level (mean $21.35 \mathrm{~g} / \mathrm{dl}$ with OR 3.4) was also found to be associated with DILI $(p<0.001)$. Forty-six patients (48.4\%) had a history of alcohol consumption and this increased the risk of DILI (OR 2.1, $p<0.002$ ).

Serological evidence confirmed recent acquisition of acute viral hepatitis in 13 patients, of whom one (1.1\%) had hepatitis A virus
(HAV) and 12 (12.6\%) had hepatitis B virus (HBV). All 13 cases of viral hepatitis were on one or more hepatotoxins identified in the study and thus were not excluded (Tables 2-5).

This study used the reference range of albumin level $<35 \mathrm{~g} / \mathrm{l}$ (NHLS normal range 35-52) to describe malnutrition. Renal disease was defined as glomerular filtration rate (GFR) $<60 \mathrm{ml} / \mathrm{min} / 1.73 \mathrm{~m}^{2}$ by Essential Drug List for Hospitals (Table 6).

This study found the top 10 drugs in decreasing order of frequency used were ART $(86.1 \%)$, alcohol $(48.4 \%)$, paracetamol $(46 \%)$, TBT $(42.1 \%)$, cotrimoxazole (32\%), traditional medication (27\%), fluconazole (14\%), amoxicillin/clavulanic acid (14\%), isoniazid (12.6\%) and statins (11.6\%). There was obvious overlap with patients taking multiple medications. A total of 63 patients $(66 \%)$ were on four or more hepatotoxic agents on presentation.

Other investigations included abdominal ultrasound and computed tomography $(\mathrm{CT})$ of the abdomen. Abdominal ultrasound was performed on 47 patients (49\%), of whom the majority were normal $(70 \%)$. Seven $(7.4 \%)$ patients with severe drug-induced liver injury were transferred for liver biopsy to a high level of care.

There were 14 deaths with a case fatality rate of $14.7 \%$. There was a significant age difference between patients who died compared with those who did not $(52.14 \pm 16$ vs. $35.6 \pm 11.6$ years; $p=0.002)$. Ten of these deaths $(71 \%)$ were complicated by HIV/TB or both. Of the total deaths, 11 (78.5\%) suffered acute liver failure and 8 (57\%) were complicated by sepsis and/or renal failure.

\section{Discussion}

According to the United Nations Global statistics, 53\% (19.6 million) of all HIV-infected patients in the world are found in sub-Saharan Africa. ${ }^{10}$ This equates to approximately $35 \%$ of the population being HIV positive. ${ }^{10}$ In 2015, 73/1 000 people died from HIV/TB related causes in South Africa (SA). ${ }^{5}$ In addition, the TB/HIV co-infection rate and use of dual therapy (ARV and TB treatment) in SA follow similar trends of between $62 \%$ and $66 \%$ respectively. ${ }^{5}$ In contrast, this study found a $37 \%$ HIV/TB coinfection rate whilst $26.3 \%$ of our patients were on dual therapy.

Worldwide the annual incidence of DILI approximates 15/100 000 persons exposed to prescription medication. ${ }^{11}$ In the United States, according to the Drug-Induced Liver Injury Network (DILIN), DILI is relatively uncommon, with an estimated frequency ranging from 1/10 000-1/100 000 patient-years of exposure. ${ }^{12}$ Our study calculated the risk of developing DILI in our population to be slightly higher at 19/100 000 .

There were more female than male patients admitted with DILI $(62.1 \%)$ and this finding is supported in other literature on gender and DILI. ${ }^{12}$ Some studies explained that this could possibly be due to nevirapine (NVP) toxicity or the increased prevalence of autoimmune hepatitis amongst women. ${ }^{13}$

International registers also showed a mean age of 55 years in the Western world and 39 years in the developing world, whilst at this site the age averaged 38 years, following a similar trend to that in India. ${ }^{12,14-16}$

In the USA, antibiotics (45\%) account for the highest cause for DILI, with antiepileptics coming in at second (15\%) and analgesics third at $5 \%{ }^{12}$ The drug distribution is slightly different for acute liver failure (ALF) as per the US ALF Group Registry Statistics 
Table 1: Patient and laboratory characteristics at baseline of those who developed drug-induced liver injury $(n=95)$

\begin{tabular}{|c|c|c|c|c|}
\hline Variable & Mean \pm SD (\%) & Minimum & Maximum & $95 \% \mathrm{Cl}$ \\
\hline Age (years) & $38.04 \pm 13.58$ & 19 & 85 & $35.27-40.81$ \\
\hline Median & 35 & & & \\
\hline \multicolumn{5}{|l|}{ Age groups: } \\
\hline $18-24$ & $9(9.5)$ & & & \\
\hline $25-29$ & $23(24.2)$ & & & \\
\hline $30-34$ & $14(14.7)$ & & & \\
\hline $35-39$ & 17 (17.9) & & & \\
\hline $40-49$ & $16(16.8)$ & & & \\
\hline $50-59$ & $6(6.3)$ & & & \\
\hline$\geq 60$ & $10(10.5)$ & & & \\
\hline \multicolumn{5}{|l|}{ Ethnicity: } \\
\hline Black & $78(82.1)$ & & & \\
\hline Indian & $11(11.6)$ & & & \\
\hline Coloured & $4(4.2)$ & & & \\
\hline White & $2(2.1)$ & & & \\
\hline \multicolumn{5}{|l|}{ Gender: } \\
\hline Male & $36(37.9)$ & & & \\
\hline Female & $59(62.1)$ & & & \\
\hline \multicolumn{5}{|l|}{ Marital status: } \\
\hline Married & $23(24.2)$ & & & \\
\hline Single & $24(25.3)$ & & & \\
\hline In a relationship & $45(47.4)$ & & & \\
\hline Divorced & $1(1.1)$ & & & \\
\hline Not documented & $2(2.1)$ & & & \\
\hline \multicolumn{5}{|l|}{ Professional status: } \\
\hline Employed & $22(24)$ & & & \\
\hline Unemployed & $73(76)$ & & & \\
\hline Weight (kg) & $56.69 \pm 9.05$ & 31 & 87 & $54.85-58.54$ \\
\hline Median & 56 & & & \\
\hline Hospital stay (days) & $8.35 \pm 4.4$ & 1 & 29 & $7.44-9.25$ \\
\hline Median & 7 & & & \\
\hline \multicolumn{5}{|l|}{ HIV status: } \\
\hline Positive & $72(75.8)$ & & & \\
\hline Negative & $23(24.2)$ & & & \\
\hline CD4 count (cells $/ \mathrm{mm}^{3}$ ) & $288.42 \pm 296.29$ & 2 & 479 & 218.79-358.04 \\
\hline Median: & 180 & & & \\
\hline$<500$ & $56(77.8)$ & & & \\
\hline$>500$ & $16(22.2)$ & & & \\
\hline \multicolumn{5}{|l|}{ Antiretroviral therapy: } \\
\hline Yes & $62(86.1)$ & & & \\
\hline No & $10(13.9)$ & & & \\
\hline \multicolumn{5}{|l|}{ Type of ART: } \\
\hline FDC & $55(89)$ & & & \\
\hline Second line & $7(11)$ & & & \\
\hline \multicolumn{5}{|l|}{ TB: } \\
\hline Positive & $41(43)$ & & & \\
\hline Negative & $54(57)$ & & & \\
\hline \multicolumn{5}{|l|}{ TB therapy: } \\
\hline Yes & $40(98)$ & & & \\
\hline No & $1(2)$ & & & \\
\hline \multicolumn{5}{|l|}{ Hepatitis screen: } \\
\hline Negative & $81(86)$ & & & \\
\hline HAV (Hep A IgM positive*) & $1(1)$ & & & \\
\hline HBV (Hep B SAg positive ${ }^{\#}$ ) & $12(13)$ & & & \\
\hline
\end{tabular}

*Hepatitis A virus: hepatitis A Immunoglobulin M.

\#Hepatitis B virus: hepatitis B Surface Antigen. 
Table 2: Multivariate analysis of risk factors for drug induced liver injury $(n=95)$

\begin{tabular}{lccc}
\hline Factor & OR & $95 \% \mathrm{Cl}$ & $p$-value \\
\hline Dependent variables: & 2.2 & $1.5-4.6$ & 0.01 \\
ALT & & & \\
\hline Independent variables: & 1.8 & $1.2-2.5$ & 0.02 \\
\hline Female gender & 3.4 & $1.8-6.1$ & 0.001 \\
Low albumin & 2.1 & $1.8-4.9$ & 0.002 \\
Alcohol & 2.6 & $2.1-6.6$ & 0.01 \\
\hline Age $>24$ years & & & \\
\hline
\end{tabular}

Table 3: Subgroup analysis of patients with drug-induced liver injury

\begin{tabular}{lcc}
\hline Subgroup & Number & Percentage \\
\hline HIV/TB naive & 18 & 18.9 \\
HIV & 37 & 38.9 \\
TB & 5 & 5.3 \\
HIV and TB & 35 & 36.8 \\
\hline
\end{tabular}

1998-2008. ${ }^{17}$ In the US ALF Registry, paracetamol is highest at 46\%, hepatitis B infection (12\%) and autoimmune conditions (5.9\%). ${ }^{17}$ ALF is largely uncommon with a prevalence of $2000-2300$ cases per annum. ${ }^{17}$ We defined ALF as INR $>/=2$, and or TBR $>200 \mathrm{mg} / \mathrm{dl}$, and hepatic decompensation (encephalopathy). ${ }^{2}$ We observed ALF in 49 patients (51\%), which similarly showed how uncommon this condition is in our setting.

Polypharmacy in our study was a problem as $66.5 \%$ of patients were taking four or more drugs on presentation. This made it difficult to pinpoint the actual causative agent.

In Japan a similar DILI distribution to that in the USA is observed; however, dietary and herbal drugs are found to be at increased frequency (17\%). ${ }^{12}$ The use of traditional medication in South Africa as a cause of DILI is largely unknown. Our study found some association with traditional medication, as we found use at $27 \%$. In India, TBT features as the top causal agent (58\%), then anti-epileptics (11\%) and lastly dapsone (5.4\%). ${ }^{14}$ Our study showed antibiotics (amoxicillin/clavulanic acid) to feature lower down on the list even after traditional medication.

Several studies have demonstrated an important association between chronic hepatitis $B / C$ infection and the development of DILI post ARV initiation. ${ }^{6,13,14}$ Wong et al. found hepatotoxicity following anti-TB drugs in 35\% of HBV carriers in contrast to $9.4 \%$
Table 5: Co-morbidity

\begin{tabular}{lcc}
\hline Co-morbidity & Number & Percentage \\
\hline Renal disease $(\mathrm{GFR}<60)$ & 33 & 34.7 \\
Malnutrition $(\mathrm{Alb}<35)$ & 30 & 31.6 \\
Hypertension & 12 & 12.6 \\
Hypercholesterolaemia & 11 & 11.6 \\
\hline Diabetes & 6 & 6.3 \\
\hline
\end{tabular}

Table 6: Exposure to various hepatotoxic drugs (excluding ART, TBT and Alcohol)

\begin{tabular}{lc}
\hline Drug & Number (\%) \\
\hline Paracetamol & $44(46.3)$ \\
\hline Cotrimoxazole & $31(32.6)$ \\
\hline Traditional medicines & $26(27.4)$ \\
\hline Fluconazole & $14(14.7)$ \\
\hline Amoxicillin/clavulanic acid & $4(14.7)$ \\
\hline Isoniazid & $12(12.6)$ \\
\hline Statins & $11(11.6)$ \\
\hline Erythromycin/azithromycin & $9(9.5)$ \\
\hline Recreational drugs & $8(8.4)$ \\
\hline Salicylates & $6(6.3)$ \\
\hline Oral hypoglycaemic drugs & $5(5.3)$ \\
\hline Oral contraceptive agents & $5(5.3)$ \\
\hline Sodium valproate & $4(4.2)$ \\
\hline Carbamazepine & $2(2.1)$ \\
\hline
\end{tabular}

non-carriers $(p<0.001) .^{18}$ In our setting we found $12.6 \%$ of DILI patients to have acute HBV infection, all of whom were on one or more hepatotoxic drugs.

Patients with malnutrition lack glutathione stores, hence their ability to clear toxic metabolites is greatly impaired. ${ }^{13,14}$ Fontana et al. found that reduced serum albumin-less than $35 \mathrm{mg} / \mathrm{ml}$ (a surrogate marker for malnutrition) - to be an independent risk factor for the development of hepatocyte death and liver transplantation. ${ }^{16}$ These findings correlate well with our research, which showed a hypoalbuminaemia (mean $21 \mathrm{mg} / \mathrm{ml}$ ) to be strongly associated with DILI $(p<0.001)$.

Current literature describes the link between anti-TB treatment and DILI. Direct toxicity has been found with isoniazid (INH) and pyrazinamide (PZA). The major drug-metabolising enzyme of INH is N-acetyltransferase2 (NAT2). ${ }^{19,20}$ Other possible enzymes

Table 4: Characteristics of patients in the four subgroups

\begin{tabular}{|c|c|c|c|c|c|}
\hline Variable & All $(n=95)$ & $\begin{array}{l}\text { TB and HIV naive } \\
\quad(n=18)\end{array}$ & $\begin{array}{c}\text { HIV } \\
(n=37)\end{array}$ & $\begin{array}{c}\text { TB } \\
(n=5)\end{array}$ & $\begin{array}{l}\text { HIV and TB } \\
\quad(n=35)\end{array}$ \\
\hline Age (years) & $38.04 \pm 13.58$ & $43.22 \pm 18.42$ & $35.86 \pm 10.59$ & $44.20 \pm 18.54$ & $36.80 \pm 12.45$ \\
\hline \multicolumn{6}{|l|}{ Gender: } \\
\hline Male & 36 & 12 & 10 & 3 & 11 \\
\hline Female & 59 & 6 & 27 & 2 & 24 \\
\hline ALT (IU/I) & $540.94 \pm 380.57$ & $494.22 \pm 394.55$ & $521.54 \pm 313.01$ & $431.00 \pm 267.53$ & $601.17 \pm 450.93$ \\
\hline Bilirubin $(\mu \mathrm{mol} / \mathrm{l})$ & $148.28 \pm 119.58$ & $173.28 \pm 176.93$ & $165.24 \pm 120.97$ & $62.00 \pm 32.50$ & $129.83 \pm 79.97$ \\
\hline Albumin (g/l) & $21.06 \pm 14.05$ & $30.00 \pm 28.07$ & $19.64 \pm 5.85$ & $27.00 \pm 5.09$ & $17.09 \pm 6.48$ \\
\hline INR & $2.20 \pm 1.20$ & $1.57 \pm 0.33$ & $2.14 \pm 1.22$ & $1.37 \pm 0.18$ & $2.69 \pm 1.34$ \\
\hline Hospital stay (days) & $8.35 \pm 4.4$ & $7.2 \pm 4.9$ & $7.6 \pm 3.6$ & $7.2 \pm 1.9$ & $9.9 \pm 4.9$ \\
\hline
\end{tabular}


include CYP2E1 and glutathione S-transferase. Abnormalities in the genes that encode for these enzymes (especially for NAT2) may predispose to problems in enzyme metabolism of drugs. NAT2 has been extensively studied and has shown that slow acetylator phenotype will increase risk of DILI when patients are exposed to $\mathrm{INH}$, and some sulphonamides. ${ }^{13,14,19,20}$ Huang showed that NAT2 slow acetylators (CYP2E1 c1/c1 genotype) were at significantly higher risk of isoniazid hepatotoxicity than rapid acetylators (CYP2E1 c1/c2 or c2/c2 genotypes; OR, 7.43). ${ }^{19}$ In developing countries where finances are scarce, this form of DILI identification might prove difficult.

Mitchell et al. demonstrated a high mortality risk (up to 50\%) in patients with DILI who present with jaundice. ${ }^{21} \mathrm{Hy}$ 's Law demonstrates a $10 \%$ or greater risk of mortality in patients with DILI who have jaundice, ALT greater than three times the upper limit of normal (ULN) or bilirubin greater than two times the ULN. ${ }^{13}$ Our study showed a $14.7 \%$ case fatality rate, of which the majority (78\%) were complicated by HIV/TB. Whether the deaths were all-cause fatality or drug-induced liver injury was unknown from this retrospective study. Other studies have shown that the prognosis of DILI is usually poorer when deranged liver function is associated with jaundice, ascites, encephalopathy and coagulopathy. ${ }^{16,22,23}$ Our study similarly showed that, of those who died, $100 \%$ had jaundice, $78.5 \%$ had encephalopathy and $50 \%$ had INR $>2$. Clinicians should thus be wary when patients with jaundice present with signs and symptoms of ALF as the prognosis is guarded and the mortality is high.

A study undertaken in Cape Town at a tertiary-level hospital (Groote Schuur) showed a mortality rate of $27 \%$ in ART-associated DILI and 35\% in TBT-associated DILI patients. ${ }^{8}$ Our study showed an overall lower mortality rate (14.7\%) and we did not draw conclusions on the causative drug due to the retrospective nature of this study. The higher mortality rate seen at Groote Schuur is in keeping with the referral of more ill patients who require that level of care. It would be interesting if a similar study were conducted in a tertiary-level hospital in KZN to compare these statistics. Policy planners at NDH should expect the admission of approximately four DILI patients per month. These patients require careful investigation and early referral as the death rate approximates 1 in 6.7 DILI admissions as demonstrated at this study site.

\section{Limitation of study}

The data collection method was retrospective and relied on the accuracy of data recorded, thus there is the possibility that patients who were not diagnosed correctly were excluded from the study.

The quantity of alcohol and traditional medication used by the patients could not be ascertained from the records.

Due to the patient numbers and descriptive nature of this study, it corresponds more to a case series. It may be useful to follow a cohort of patients with jaundice/DILI from admission to diagnosis to more fully understand and document the risk factors and mortality of DILI at the study site.

\section{Recommendations}

A high index of suspicion should be maintained at all levels of care in patients with jaundice who have risks associated with DILI. As record keeping is largely paper based at this site, and driven by junior staff in the admissions area, an easy-to-use tick sheet is thus advocated in the identification of high-risk
DILI patients and to identify all new and old medication being taken.

This study was carried out at one study site and findings may be strengthened by expanding the study to other sites.

\section{Conclusions}

This study showed possible associations with DILI and being female, younger age group (24-38 years), hypoalbuminaemia and renal failure. However, the study design does not allow us to make any direct associations and further studies with more appropriate research designs are recommended. The use of alcohol, traditional medication and the excessive use of paracetamol in patients who present with DILI is very concerning. The case fatality rate of $14.7 \%$ demonstrates the importance of identifying these potential risk factors timeously.

The use of ARV and TBT has shown a positive association with DILI, especially in the first three months of treatment. This correlates with current knowledge. The appropriate suspicion, screening and clinical reasoning should be undertaken in any patient who presents with illness within the first three months of starting a new drug.

Disclosure statement - No potential conflict of interest was reported by the authors.

\section{References}

1. Menezes C. An approach to the management of Drug-induced liver injury in HIV- infected patients treated for Tuberculosis. Department of Internal Medicine (Power Point Slides 1-43) [cited 2019 Jan 23]. Available from https://sahivsoc.org

2. Wang $S$, Shangguan $Y$, Ding $C$, et al. Risk factors for acute liver failure among inpatients with anti-tuberculosis drug-induced liver injury. J Int Med Res. November 26, 2018. [cited 2019 Jan 23]. https://doi. org/10.1177/0300060518811512

3. Wood R, Bekker LG. Isoniazid preventive therapy for tuberculosis in South Africa: An assessment of the local evidence base. SAMJ. 2014;104(3):174-177 [cited 2017 Jan 13].

4. Department of Health. KZN Department of Health Implements HIV 'Test and Treat' Policy Media Relase: 01 September 2016 [cited 2016 Dec 12]. Available from www.kznhealth.gov.za

5. The Global Tuberculosis report. 2016 [cited 2017 Mar 18]. Available from: www.who.int/tb/data

6. Jong E, Conradie F, Berhanu R, et al. Consensus statement: Management of drug-induced liver injury in HIV-positive patients treated for TB. South Afr J HIV Med. 2013;14(3):113-119.

7. Cohen $K$, Meintjes G. Management of individuals requiring ART and TB treatment. Curr Opin HIV AIDS. 2010 Jan;5(1):61-69 [cited 2017 Apr 21]. Available from: www.ncbi.nlm.nih.gov

8. Schutz C, Ismail Z, Proxenos $\mathrm{CJ}$, et al. Burden of antituberculosis and antiretroviral drug-induced liver injury at a secondary hospital in South Africa. S Afr Med J. 2012;102(6):506-511.

9. Department of health. District health plan 2015-2016 [cited 2016 Dec 20]. Available from www.kznhealth.gov.za

10. Global HIV and AIDA statistics- 2018 fact sheet. UNAIDS Report on the AIDS epidemic Geneva UNAIDS, 2016, 2016 [cited 2019 Jan 22]. Available from www.unaids.org

11. Drug Induced Liver Injury. Up-to-date: [cited 2019 Aug 8]. Available from http//www.uaeps.me

12. Fontana $\mathrm{RJ}$, Watkins $\mathrm{PB}$, Bonkovsky $\mathrm{HL}$, et al. Drug induced liver injury network (DILIN)- prospective study. Drug Sa. 2009;32(1):55-68.

13. McGovern BH, Sulkowski MS, Sterling RK. Chapter 38 - HIV Co-infection drug toxicity. In: Sanyal TDBPMJ, editor. Zakim and Boyer's hepatology. 6th ed. Saint Louis: W.B. Saunders; 2012. p. 683-699.

14. Devarbhavi $\mathrm{H}$. An update on drug-induced liver injury. J Clin Exp Hepatol. 2012 9;2(3):247-259. 
15. Vuppalanchi R, Chalasani N. Chapter 16 - Risk factors for druginduced liver disease. In: DeLeve NKD, editor. Drug-induced liver disease. 3rd ed. Boston: Academic Press; 2013. p. 265-274.

16. Fontana RJ, Hayashi PH, Gu J, et al. Idiosyncratic drug-induced liver injury is associated with substantial morbidity and mortality within 6 Months from onset. Gastroenterology. 2014;147 (1):96-108.e4

17. Torres DM, Stevens RD, Gurakar A. Acute liver failure, a management challenge for the practicing Gastroenterlogist. Gastroenterol Hepatol (NY). 2010 Jul;6(7):444-450.

18. Wong WM, Wu PC, Yuen MF, et al. Antituberculosis drug- related liver dysfunction in chronic hepatitis B. Hepatology. 2000;31:201-206.

19. Huang Y-S. Recent progress in genetic variation and risk of antituberculosis drug-induced liver injury. J Chin Med Assoc. 2014;77(4):169-173.
20. Singla N, Gupta D, Birbian N, et al. Association of NAT2. GST and CY: 2E, 1 polymorphisms and anti-tuberculosis drug-induced hepatotoxicity. Tuberculosis. 2014;94(3):293-298.

21. Mitchell JR, Zimmerman HJ, Ishak KG, et al. Isoniazid liver injury: clinical spectrum, pathology, and probable pathogenesis. Ann Intern Med. 1976 Feb;84(2):181-192.

22. Chalasani N, Fontana RJ, Bonkovsky HL, et al. Causes, clinical features, and outcomes from a prospective study of drug-induced liver injury in the United States. Gastroenterology. 2008 Dec;135(6):1924-1934. 34 e1-4. PubMed PMID: 18955056. Pubmed Central PMCID: PMC3654244. Epub 2008/10/29. eng.

23. Regev A, Björnsson ES. Drug-induced liver injury: morbidity, mortality, and Hy's law. Gastroenterology. 2014;147(1):20-24.

Received: 22-05-2019 Accepted: 15-08-2019 